\title{
Proton Spectroscopy Detected Myoinositol in Children with Traumatic Brain Injury
}

\author{
STEPHEN ASHWAL, BARBARA HOLSHOUSER, KAREN TONG, TERESA SERNA, \\ RENATTA OSTERDOCK, MATTHEW GROSS, AND DANIEL KIDO
}

\author{
Division of Child Neurology, Department of Pediatrics [SA, TS]; Department of Radiology [BH, KT, DK]; \\ Department of Neurosurgery [RO]; Division of Critical Care Medicine, Department of Pediatrics [MG], \\ Loma Linda University School of Medicine, Loma Linda, CA 92350
}

\begin{abstract}
Previous studies have shown that proton magnetic resonance spectroscopy (MRS) is useful in predicting neurologic prognosis in children with traumatic brain injury (TBI). Reductions in $\mathrm{N}$-acetyl derived metabolites and presence of lactate have been predictive of poor outcomes. We examined another spectroscopy metabolite, myoinositol (mI), to determine whether it is altered after TBI. Found primarily in astrocytes, $\mathrm{mI}$ functions as an osmolyte and is involved in hormone response pathways and protein-kinase $\mathrm{C}$ activation. Myoinositol is elevated in the newborn brain and is increased in a variety of diseases. We studied 38 children (mean age $11 \mathrm{y}$; range 1.6-17 y) with TBI using quantitative short echo time occipital gray and parietal white matter proton MRS at a mean of $7 \mathrm{~d}$ (range 1-17 d) after injury. We found that occipital gray matter $\mathrm{mI}$ levels were increased in children with TBI $(4.30 \pm 0.73)$ compared with controls $(3.53 \pm 0.48 ; p=$ $0.003)$. We also found that patients with poor outcomes $6-12$ mo after injury had higher $\mathrm{mI}$ levels $(4.78 \pm 0.68)$ than patients with good outcomes $(4.15 \pm 0.69 ; p<0.05)$. Myoinositol is elevated after pediatric TBI and is associated with a poor neurologic outcome. The reasons for its elevation remain unclear but may be due to astrogliosis or to a disturbance in osmotic function. (Pediatr Res 56: 630-638, 2004)
\end{abstract}

\section{ABSTRACT}

Cho, choline

Cre, creatine

DAI, diffuse axonal injury

GCS, Glasgow Coma Scale

GOS, Glasgow Outcome Scale

HMIT, H+/myo-inositol symporter

ICP, intracranial pressure

IP3, inositol triphosphate

MAP, mean arterial blood pressure

mI, myoinositol

MRI, magnetic resonance imaging

MRS, magnetic resonance spectroscopy

NAA, N-acetyl aspartate

NAAG, N-acetylaspartylglutamate

OGM, occipital gray matter

PCPCS, Pediatric Cerebral Category Performance Scale

PVS, persistent vegetative state

PWM, parieto-occipital white matter

SMIT, sodium-mI transporter

STEAM, stimulated echo acquisition mode

SWI, susceptibility weighted imaging

TBI, traumatic brain injury
Over the past decade, proton magnetic resonance spectroscopy (MRS) has been proven helpful in evaluating adults and children with traumatic brain injury (TBI) $(1,2)$. Reductions in the neuronal marker N-acetyl aspartate (NAA) and in creatine (Cre), a marker of cell energy metabolism, as well as increases in choline (Cho), an indicator of membrane disruption, occur within the first several days. In adults, the magnitude of these metabolite changes correlate with the severity of injury and also with neurologic and neuropsychological outcomes $(3,4)$. Our previous studies in children have also confirmed these findings $(5,6)$.

Received January13, 2004; Accepted June 22, 2004.

Correspondence: Stephen Ashwal, M.D., Department of Pediatrics, Loma Linda University School of Medicine,11175 Campus Street, Room A1120G, Loma Linda, CA 92350; e-mail: sashwal@ahs.llumc.edu

DOI: 10.1203/01.PDR.0000139928.60530.7D
Myoinositol (mI) is another metabolite that can be detected using short echo time spectroscopy. Myoinositol is found primarily in astrocytes and functions as a marker of astrocytic activity. It serves as an osmolyte, and is involved in phosphoinositide-mediated signal transduction. Myoinositol as detected by MRS has been shown to be elevated in the newborn brain and is increased in Alzheimer's disease, renal failure, diabetes mellitus, bipolar disease, use of lithium, hypoxia, hyperosmolar states, in some patients with Canavan disease and after severe neonatal encephalopathy $(7,8)$. It may be decreased in chronic hepatic encephalopathies, stroke, tumor, infection and low-grade malignancies.

Within the first hours after TBI, the complex process referred to as reactive astrocytosis, astrogliosis, or glial scarring begins (9). After the initial injury, which is accompanied by 
neuronal and glial cell death, followed later by apoptotic cell loss, recruitment of microglia and peripheral monocytes occurs. Nestin-positive progenitor cells differentiate into astrocytes at the site of injury and within 3 to $5 \mathrm{~d}$ the process of reactive astrocytosis begins to surround the area of injury. While it remains controversial whether the process of astrogliosis that results in a glial scar is ultimately beneficial or detrimental to the degree of cerebral recovery, it is clear that astrocytes play an important role in the evolution of this process both in the mature and developing nervous system $(9,10)$.

In the current study we examined the role of $\mathrm{mI}$ in 38 children with TBI. These patients also all had evidence of diffuse axonal injury (DAI) detected by a new gradient echo magnetic resonance imaging (MRI) technique known as susceptibility weighted imaging (SWI) recently found useful in detecting hemorrhagic lesions associated with DAI (11). We were interested in determining if children with DAI had alterations in $\mathrm{mI}$, as a possible marker of astrocytic activity or as a marker of altered osmolar function and whether this correlated with clinical and spectroscopy variables as well as $6-12$ mo neurologic outcomes. We were also interested in determining if there was a relation between changes in the spectral metabolite Glx (glutamate/glutamine) as studies in adult TBI patients $(12,13)$ and in children with nonaccidental trauma (14) have shown that glutamate as measured in ventricular cerebrospinal fluid by microdialysis is increased.

\section{METHODS}

Patient selection and data collection. We studied 38 children and adolescents with TBI and presumed DAI admitted to the pediatric or adult intensive care units at Loma Linda University Medical Center between March, 2001 and September, 2002. The study population was a nonconsecutive series of patients with a well-documented history of TBI who were clinically suspected to be at high risk for long-term neurologic impairment. Patients were considered at high risk if they did not show significant recovery within the first day after admission. We excluded children with a history of known CNS malformation, developmental disability, previous brain injury, or age younger than one month. A neurosurgeon or neurologist examined all children before the MRI study. These children were also part of a larger ongoing study, examining the role of magnetic resonance spectroscopy (MRS) for outcome prediction in children and adolescents with various forms of acute CNS insults, which was approved by the Institutional Review Board of Loma Linda University Medical Center.

Clinical variables determined included age, initial Glasgow Coma Scale (GCS) score, first determined mean arterial blood pressure (MAP), heart rate, arterial blood $\mathrm{pH}$, hematocrit, serum glucose and sodium levels, occurrence of cardiac arrest or presence of fixed dilated pupils, initial intracranial pressure (ICP) level if monitored, occurrence of seizures, days in coma, on ventilator, and in hospital, and discharge outcome and outcome 6 to 12 mo after injury as determined by the Pediatric Cerebral Category Performance Scale score (15). The PCPCS is a six-point outcome scoring system modified from the
Glasgow Outcome Scale (GOS) score. It quantifies the overall functional morbidity and cognitive impairment of infants and children, has been validated in pediatric patients after acute CNS injuries and recently has been shown to correlate with other measures of psychometric function (16). The score includes the following outcomes: (1) normal: can perform all age-appropriate activities; (2) mild disability: conscious, alert and able to interact at most age-appropriate activities and may have mild neurologic deficit; (3) moderate disability: conscious, sufficient cerebral function for age-appropriate activities of daily life, but has significant cognitive impairment; (4) severe disability: conscious, dependent on others for daily support because of impaired brain function; (5) persistent vegetative state (PVS); and (6) death. For the purpose of analysis, the six PCPCS scores were dichotomized into two groups: (1) normal or mild disability and (2) moderate, severe disability or vegetative state. There were no patients who died.

A group of 10 control patients seen during the same time period of the study were used so that we could compare the MRS results of the TBI patients with normative data. Three controls were normal volunteers who did not require sedation. The other controls were patients who had neuroimaging done for evaluation of clinical neurologic or craniofacial disorders (e.g. headaches, facial hemangiomas, isolated seizure, etc) and were neurologically normal on examination.

MR imaging. When medically stable, children were transported to the MR scanner and were monitored during these studies by intensive care unit and radiology personnel. All studies were performed on a $1.5 \mathrm{~T}$ whole body imaging system using a standard quadrature head coil (Siemens Medical Solutions, Vision, Numaris VB33D, Iselin, NJ).

MRI sequences included sagittal T1 weighted scans (TR/TE $=500 / 14 \mathrm{~ms}, 5 \mathrm{~mm}$ thick, $20 \%$ gap), axial and coronal fast $\mathrm{T} 2$ weighted scans (TR/TE $=3500 / 90 \mathrm{~ms}, 5 \mathrm{~mm}$ thick/ 40\% gap), and axial 3D gradient echo susceptibility weighted scans $\left(\mathrm{TR} / \mathrm{TE}=57 / 40 \mathrm{~ms}\right.$, flip angle $=20^{\circ}, 32$ partitions of $2 \mathrm{~mm}$ thick). These scans were used for comparison with the information available on SWI images. The SWI sequence consisted of a strongly susceptibility-weighted, low-bandwidth $(78 \mathrm{~Hz} /$ pixel) $3 \mathrm{D}-\mathrm{FLASH}$ sequence $\left(\mathrm{TR} / \mathrm{TE}=57 / 40 \mathrm{~ms}, \mathrm{FA}=20^{\circ}\right)$ with first-order flow compensated in all 3 orthogonal directions. Thirty-two partitions of $2 \mathrm{~mm}$ were acquired using a rectangular FOV $(5 / 8$ of $256 \mathrm{~mm})$ and a matrix size of $160 \times$ 512 , resulting in a voxel size of $1 \times 0.5 \times 2 \mathrm{~mm}^{3}$. The SWI sequence included the majority of the cerebral hemispheres and the posterior fossa, with an acquisition time of approximately 9.5 min. SWI images were created using the magnitude and phase images as previously described $(11,17)$. Hemorrhagic lesions were defined as hypointense foci that were not compatible with vascular, bony or artifactual structures on conventional GRE or SWI. Hemorrhagic shearing lesions of the entire cranium were counted individually on SWI sequences. Because hemorrhagic lesions were variable in shape, images were then analyzed using a computer software program (Image Pro Plus, Media Cybernetics Inc.) to automatically count the number and calculate the area and volume of lesions (expressed as $\mathrm{mm}^{3}$ ). 
Table 1. Comparison of clinical variables between dichotomized outcome groups

\begin{tabular}{|c|c|c|c|}
\hline Variables & $\begin{array}{l}\text { Good Outcome* } \\
\quad(n=29)\end{array}$ & $\begin{array}{l}\text { Poor Outcome* } \\
\quad(n=9)\end{array}$ & $p$ value \\
\hline Age (years) & $11.9 \pm 5.9$ & $10 \pm 3.0$ & 0.22 \\
\hline GCS & $7 \pm 4$ & $4.0 \pm 1.0$ & $0.03 \dagger$ \\
\hline Days on ventilator & $6 \pm 6$ & $11 \pm 6$ & $0.02 \dagger$ \\
\hline Days in hospital & $27 \pm 25$ & $43 \pm 22$ & 0.06 \\
\hline Days to MRS & $7 \pm 4$ & $8 \pm 4$ & 0.36 \\
\hline Serum glucose —admission (mg/dl) & $189 \pm 77$ & $149 \pm 42$ & 0.06 \\
\hline Serum glucose-day of MRS (mg/dl) & $114 \pm 21$ & $114 \pm 11$ & 0.99 \\
\hline Cerebral perfusion pressure - initial $(\mathrm{mmHg})$ & $63 \pm 19$ & $50 \pm 23$ & 0.19 \\
\hline Total \# of hemorrhagic lesions & $116 \pm 148$ & $320 \pm 202$ & $0.003 \dagger$ \\
\hline Total volume of hemorrhagic lesions $\left(\mathrm{mm}^{3}\right)$ & $11519 \pm 15949$ & $38714 \pm 32632$ & $0.003 \dagger$ \\
\hline
\end{tabular}

* Good Outcome, Normal, or Mild Disability; Poor Outcome, Moderate, Severe Disability or Vegetative State.

$\dagger$ Mann-Whitney Test; $p \leq 0.05$ considered significant.

MR Spectroscopy. Two single voxel proton spectra were acquired with $8 \mathrm{~mL}$ volumes in normal appearing brain; one in the occipital gray matter (OGM) located in a paramedian position across the interhemispheric fissure and a second in the parieto-occipital white matter (PWM) placed in the right or left hemisphere to avoid obvious areas of injury. Previous studies have shown that measurements from these locations produce accurate long-term outcome predictions $(2,3,18)$. To predict global injury, we felt that it was important not to acquire spectra from brain with obvious hemorrhage or contusion because larger spectral changes produced in these focal areas of injury would tend to overestimate the total amount of injury and ultimately produce false positive predictions. A watersuppressed stimulated echo acquisition mode (STEAM) sequence was used with TR/TE/TM $=3000 / 20 / 13 \mathrm{~ms}$ and 128 NEX, following manual localized shimming to achieve water line widths less than $8 \mathrm{~Hz}$. A reference spectrum was acquired with identical acquisition parameters and 8 acquisitions to use for eddy current correction. Metabolite levels for NAA, Cre, Cho, mI, and Glx (glutamate/glutamine) for each of the 38 patients were quantitatively measured using a Linear Combination of Model in vitro spectra, (LCModel), an automatic (user independent), frequency-domain fitting routine. The method employs a basis set of concentration calibrated model spectra of individual metabolites to estimate absolute concentrations of similar brain metabolites from in vivo spectral data correcting for residual eddy current effects and actual coil loading by using the transmitter reference amplitude (19). This method offers several advantages. It allows for distinction between metabolites with overlapping signals to yield individual concentrations as well as providing summation data for overlapping resonances, such as NAA and N-aceylaspartylglutamate (NAAG) often seen in in vivo spectral data. In addition, LCModel estimates a baseline for short echo time spectra, which includes macromolecules with short $\mathrm{T} 2$ relaxation times making a fit of the lower concentrations and overlapping
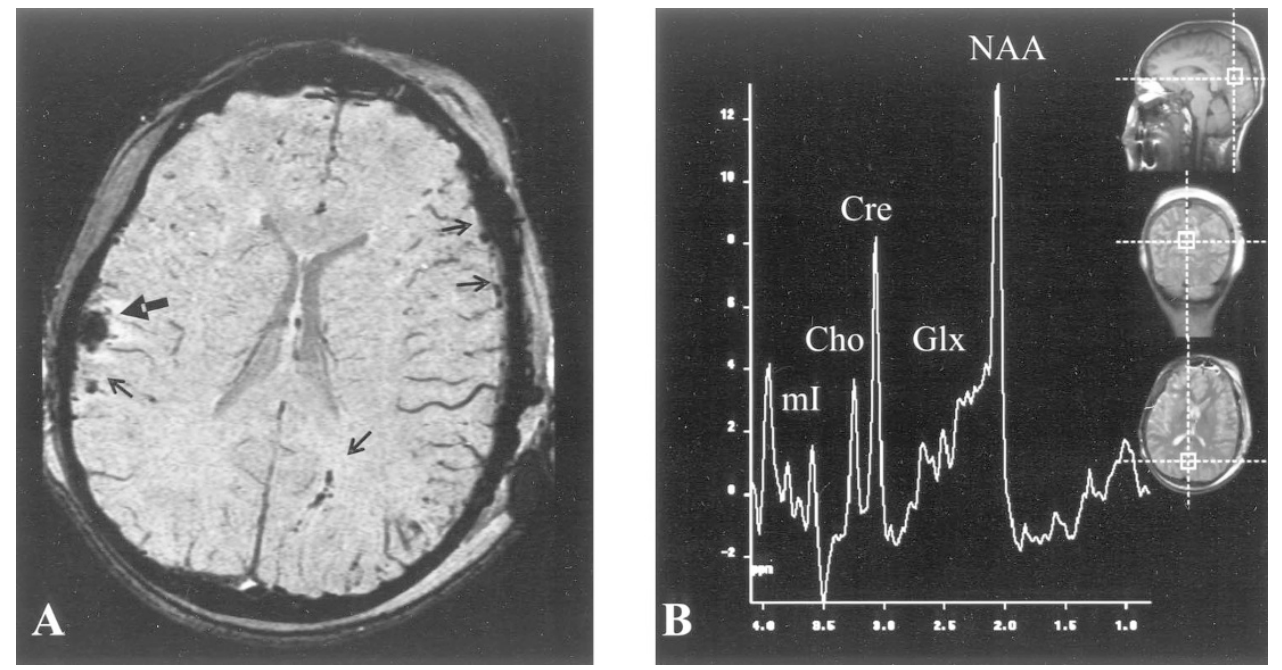

Figure 1. MRI and MRS images obtained five days after a fourteen-year old male riding a scooter was struck by a motor vehicle and had initial GCS of 14 . $\mathrm{He}$ was on ventilator support for $2 \mathrm{~d}$ and in hospital for $14 \mathrm{~d}$. Axial SWI $(A)$ images (3D-FLASH, TR/TE $=57 / 40 \mathrm{~ms}$, flip angle $=20^{\circ}$, bandwidth $=78 \mathrm{~Hz} /$ pixel) demonstrate a few scattered small hypointense lesions consistent with microhemorrhages in the cortex and subcortical white matter of the hemispheres (small arrows). A larger, approximately $1 \mathrm{~cm}$ diameter, hemorrhage with surrounding edema is seen in the periphery of the right parietal lobe (large arrow). The single voxel spectrum in the mid-occipital gray matter $(B)$ obtained at the same level shows relatively normal metabolite peaks. At 6-12 mo follow-up, he had a normal outcome score. 

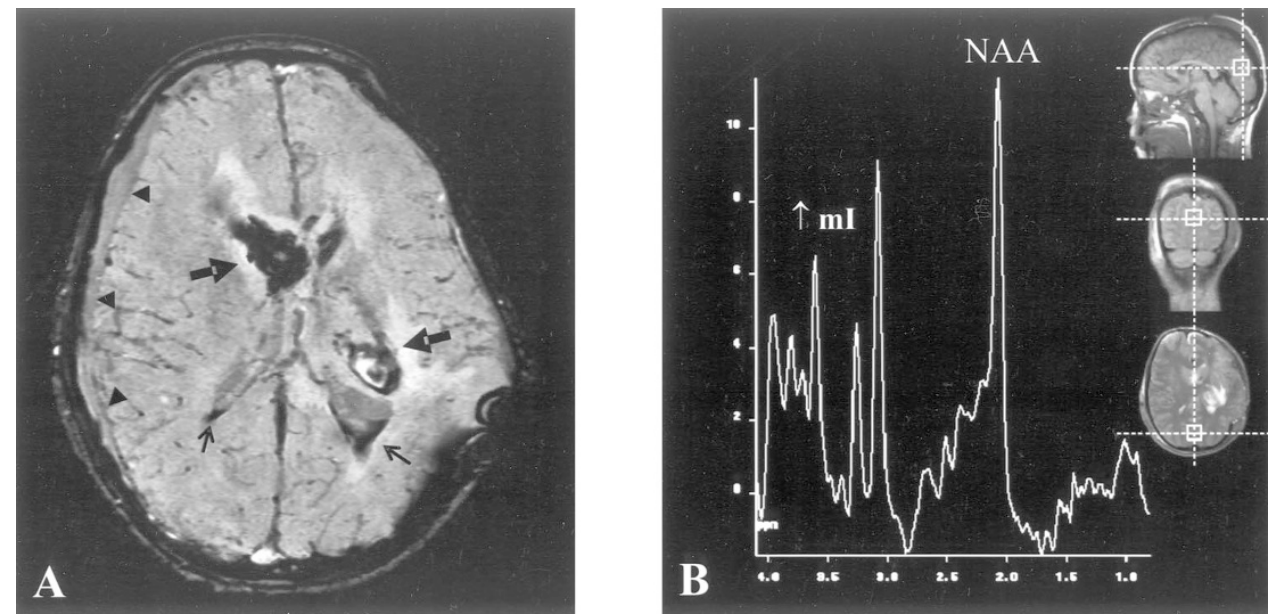

Figure 2. MRI and MRS images obtained two weeks after an eleven-year old female pedestrian was struck by a motor vehicle and had an initial GCS of 4 . She was on ventilator support for $12 \mathrm{~d}$ and in hospital for $60 \mathrm{~d}$. Axial SWI images $(A)$ demonstrate moderately large hemorrhages in the anterior corpus callosum and left thalamus (large arrows). Mild intraventricular blood (small arrows) and right subdural fluid (arrowheads) are also observed. Note that a left craniotomy has been performed for decompression. The single voxel spectrum in the mid-occipital gray matter $(B)$ obtained at the same level shows elevated myoinositol. The NAA peak is also diminished. At 6-12 mo follow-up, she had severe disability.

resonances of Glx possible and reproducible from in vivo spectra. Also each concentration is reported with a confidence measurement $(\mathrm{SD} \%)$ reflecting maximum-likelihood estimates and their uncertainties (20). Metabolite concentrations with a $\mathrm{SD} \%$ of 25 or less were included in the study. Differences between in vivo and in vitro $\mathrm{T} 2$ relaxation times were not taken into account since these corrections would be small and overly time-consuming to incorporate into a clinical study. T1 saturation effects can be ignored since long repetition times were used for all acquisitions. The following metabolite ratios were also calculated: NAA/Cre, NAA/Cho, Cho/Cre, mI/Cre and Glx/Cre.

Data analysis. For all TBI patients, we used a nonparametric Mann-Whitney test to determine significant differences between the means of good outcome patients versus poor outcome patients for clinical and imaging data using the SPSS Statistics for Windows (SPSS Inc., Release 10.0, Chicago, IL). A one-way analysis of variance (ANOVA) with a post hoc Scheffe test was used to compare spectroscopy variables in the control group to the good and poor outcome TBI groups. Differences were considered significant at $p \leq 0.05$. Correlations between $\mathrm{mI}$ and Glx in gray and white matter to clinical and other spectroscopy variables were reported as Spearman rho correlation coefficients with significance at $p \leq 0.05$. A logistic regression analysis was also performed to determine which spectroscopy variable alone or in combination with clinical variables best predicted the correct long-term dichotomous outcomes. The analysis assigns weighting factors to each variable. A large weighting factor means that the variable is strongly predictive of outcome. In this way, we were able to determine which variables were prognostic and should be used in the analysis.

\section{RESULTS}

Clinical data. Table 1 summarizes clinical data on the dichotomized outcome groups (good, $n=29$; poor, $n=9$ ). Patients with poor outcomes had lower GCS scores, a longer duration of coma and more days of ventilator support. They also had evidence of more severe DAI as manifested by a greater number and volume of hemorrhagic lesions detected by
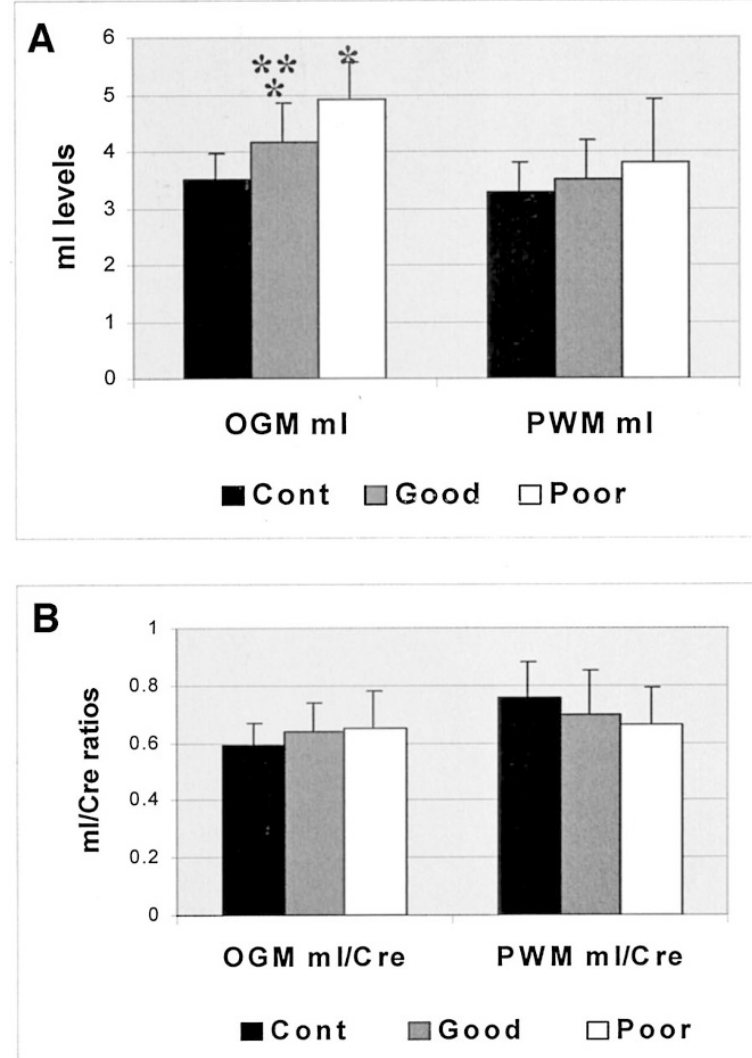

Figure 3. Quantitative metabolite data $(A)$ show that children with TBI with either good or poor $(n=38)$ outcomes had higher occipital gray matter (OGM) mI levels than controls $(n=10)\left({ }^{*} p=0.003\right)$. Also patients with poor outcomes $(n$ $=9)$ had higher OGM mI levels than those with good outcomes $(n=29)(* * p<$ 0.05 ). No differences between controls and good $v s$. poor TBI patients were seen in the parietal white matter (PWM). Results for the myoinositol/creatine (mI/creatine) ratios $(B)$ show no differences in either OGM or PWM between the three groups. 
Table 2. Metabolite levels and ratios from short echo time single voxel proton MRS in normal appearing brain

\begin{tabular}{|c|c|c|c|c|}
\hline & $\begin{array}{l}\text { Controls } \\
(n=10)\end{array}$ & $\begin{array}{l}\text { All TBI } \\
(n=38)\end{array}$ & $\begin{array}{l}\text { Good outcome } \\
\quad(n=29)\end{array}$ & $\begin{array}{l}\text { Poor outcome } \\
\quad(n=9)\end{array}$ \\
\hline \multicolumn{5}{|l|}{ Metabolite Levels } \\
\hline OGM-NAA & $9.23 \pm 0.83$ & $8.85 \pm 1.1$ & $8.92 \pm 1.2$ & $8.63 \pm 0.65$ \\
\hline OGM-Cho & $2.76 \pm 0.48$ & $3.52 \pm 0.66$ & $3.53 \pm 0.67$ & $3.50 \pm 0.67$ \\
\hline OGM-mI & $3.53 \pm 0.48$ & $4.30 \pm 0.73$ & $4.15 \pm 0.69$ & $4.78 \pm 0.68$ \\
\hline OGM-Glx & $10.7 \pm 1.8$ & $13.5 \pm 2.4$ & $13.2 \pm 2.2$ & $14.5 \pm 3.0$ \\
\hline PWM-NAA & $7.98 \pm 1.1$ & $7.83 \pm 1.6$ & $8.25 \pm 1.3$ & $6.49 \pm 1.5$ \\
\hline PWM-mI & $3.29 \pm 0.51$ & $3.52 \pm 0.77$ & $3.50 \pm 0.70$ & $3.62 \pm 1.0$ \\
\hline PWM-Glx & $7.96 \pm 2.6$ & $10.3 \pm 3.6$ & $10.1 \pm 3.2$ & $11.0 \pm 4.8$ \\
\hline \multicolumn{5}{|l|}{ Metabolite Ratios } \\
\hline OGM-NAA/Cre & $1.53 \pm 0.11$ & $1.34 \pm 0.19$ & $1.38 \pm 0.19$ & $1.21 \pm 0.14$ \\
\hline OGM-NAA/Cho & $3.43 \pm 0.65$ & $2.60 \pm 0.54$ & $2.60 \pm 0.53$ & $2.56 \pm 0.58$ \\
\hline OGM-Cho/Cre & $0.47 \pm 0.06$ & $0.53 \pm 0.10$ & $0.54 \pm 0.10$ & $0.49 \pm 0.08$ \\
\hline OGM-mI/Cre & $0.59 \pm 0.08$ & $0.65 \pm 0.10$ & $0.64 \pm 0.10$ & $0.67 \pm 0.09$ \\
\hline PWM-Glx/Cre & $1.77 \pm 0.32$ & $2.01 \pm 0.54$ & $2.03 \pm 0.57$ & $1.97 \pm 0.43$ \\
\hline
\end{tabular}

OGM, occipital gray matter; PWM, parietal white matter.

* Designates significance at $p<0.05$; ANOVA, post hoc Scheffe Test.

MRI. There were no differences between groups for the serum sodium or glucose levels measured initially or on the day of MRS. In addition, the average cerebral perfusion pressure (intracranial pressure - mean arterial pressure) for the first day of ICP monitoring was not significantly different between groups.

MRS data. Figure 1 demonstrates SWI images (A) and a MRS spectrum (B) from a patient with a good outcome who has normal metabolite and $\mathrm{mI}$ peaks. Figure 2 shows imaging and spectral data from a patient with a poor outcome who had a reduced NAA and an elevated $\mathrm{mI}$ peak.

Table 2 summarizes data for all metabolite levels and ratios in normal appearing brain. Quantitative Glx and $\mathrm{mI}$ levels in OGM were significantly higher in children who suffered TBI compared with controls. We found no significant difference in Glx for patients with good outcomes compared with those with poor outcomes, however, $\mathrm{mI}$ was significantly higher for patients with poor outcomes $(p<0.05)$ (Fig. 3A). We found no significant differences in quantitative PWM Glx or mI levels and no significant differences in metabolite ratios for either location (Table 2 and Fig. $3 B$ ).

OGM and PWM NAA/Cre and NAA/Cho ratios were lower in TBI patients compared with controls. In addition, the NAA/ Cre in both regions were lower in poor compared with good outcome patients. Based on the quantitative MRS data, these ratio changes were due to both higher Cre and to lower NAA levels (which reached significance in PWM but not OGM) that occur after TBI.

Table 3 summarizes the Spearman Rho correlation coefficients and $p$ values for selected clinical and spectroscopy values for OGM and PWM quantitative Glx and $\mathrm{mI}$ values. OGM mI levels correlated with duration of coma, days on ventilator, days in hospital, volume of SWI lesions and 6-12 mo outcome, whereas, Glx levels did not. Significant linear correlations were observed in OGM and PWM between $\mathrm{mI}$, Glx, and Cre.
Table 4 summarizes results of the logistic regression analysis. Clinical variables (GCS score, number of days in coma or on ventilator) alone or in combination correctly predicted outcome in $76 \%$ to $82 \%$ of cases, however, none of the predictions were significant. The total number and volume of hemorrhagic DAI lesions as measured with SWI correctly predicted outcome in $79 \%$ of patients and different combinations of the MRS variables predicted outcome in $79 \%$ to $82 \%$ of outcomes. Although several MRS variables alone or in combination correctly predicted a high percentage of outcomes, only a few approached significance using a $\chi^{2}$ test. For example, OGM mI alone correctly predicted outcome in $79 \%$ ( $p=0.07$ ) of patients with $1 / 29$ false positive cases (i.e., poor outcome predicted but good outcome occurred) and 6/9 false negative cases (i.e., good outcome predicted but poor outcome occurred) and OGM Glx alone predicted 79\% $(p=0.11)$ with no false positive cases. For comparison, OGM NAA and Cre predicted $76 \%(p=0.70)$ and $79 \%(p=0.39)$, respectively with $100 \%$ false negative cases for NAA. By combining several OGM MRS variables (Cre, $\mathrm{mI}$ and Glx), the predictive accuracy increased to $82 \%$ with $p=0.08$. Combining neuroimaging or clinical variables with MRS also tended to increase the number of correct predictions, but the measurements were not significant. In addition, metabolite ratios were also tested and were found to be less predictive. Therefore, it appears that using MRS levels (particularly from OGM) alone or in combination tended to produce the best results.

\section{DISCUSSION}

Myoinositol was first isolated as a chemical compound in 1850 and later found to be one of nine stereoisomers of the compound hexahydroxycyclohexane (7). Myoinositol is the most common of these stereoisomers and is conventionally inferred when the term inositol is used. In humans, $\mathrm{mI}$ content is greatest in the kidneys, brain, and testes. Dietary sources 
Table 2. Continued

\begin{tabular}{|c|c|c|c|c|}
\hline & $\begin{array}{c}p \text { value } \\
\text { (all TBI vs control) }\end{array}$ & $\begin{array}{c}p \text { value } \\
\text { (good vs control) }\end{array}$ & $\begin{array}{c}p \text { value } \\
\text { (poor vs control) }\end{array}$ & $\begin{array}{c}p \text { value } \\
\text { (good vs poor) }\end{array}$ \\
\hline \multicolumn{5}{|l|}{ Metabolite Levels } \\
\hline OGM-NAA & 0.33 & 0.74 & 0.48 & 0.77 \\
\hline OGM-Cre & $0.02 *$ & 0.19 & $0.004 *$ & 0.06 \\
\hline OGM-Cho & $0.001 *$ & $0.007^{*}$ & $0.05^{*}$ & 0.99 \\
\hline OGM-mI & $0.003 *$ & $0.04^{*}$ & $0.001^{*}$ & $0.05 *$ \\
\hline OGM-Glx & $0.002 *$ & $0.02 *$ & $0.003 *$ & 0.31 \\
\hline PWM-NAA & 0.79 & 0.88 & 0.08 & $0.005^{*}$ \\
\hline PWM-Cre & $0.06 *$ & 0.27 & 0.14 & 0.68 \\
\hline PWM-Cho & $0.005^{*}$ & $0.01 *$ & 0.29 & 0.42 \\
\hline PWM-mI & 0.42 & 0.79 & 0.66 & 0.91 \\
\hline PWM-Glx & 0.09 & 0.31 & 0.22 & 0.82 \\
\hline \multicolumn{5}{|l|}{ Metabolite Ratios } \\
\hline OGM-NAA/Cre & $0.00 *$ & $0.05^{*}$ & $0.001 *$ & $0.05 *$ \\
\hline OGM-NAA/Cho & $0.00 *$ & $0.001 *$ & $0.007 *$ & 0.98 \\
\hline OGM-Cho/Cre & $0.03 *$ & $0.04^{*}$ & 0.78 & 0.24 \\
\hline OGM-mI/Cre & 0.09 & 0.36 & 0.17 & 0.66 \\
\hline OGM-Glx/Cre & $0.003 *$ & 0.15 & 0.32 & 1.0 \\
\hline PWM-NAA/Cre & $0.03 *$ & 0.27 & $0.000 *$ & $0.002 *$ \\
\hline PWM-NAA/Cho & $0.00 *$ & $0.002 *$ & $0.000 *$ & 0.20 \\
\hline PWM-Cho/Cre & 0.24 & 0.27 & 0.95 & 0.10 \\
\hline PWM-mI/Cre & 0.25 & 0.58 & 0.52 & 0.93 \\
\hline PWM-Glx/Cre & 0.25 & 0.50 & 0.73 & 0.97 \\
\hline
\end{tabular}

OGM, occipital gray matter; PWM, parietal white matter.

* Designates significance at $p<0.05$; ANOVA, post hoc Scheffe Test.

provide about $1 \mathrm{~g} / \mathrm{d}$ of inositol. Another $4 \mathrm{~g} / \mathrm{d}$ are synthesized from glucose, primarily by the kidneys, although the brain, liver, and testes can synthesize $\mathrm{mI}$ to a lesser extent. The relatively high concentration of $\mathrm{mI}$ in the brain, as recognized in 1934, was suggestive of functional importance. It has been shown since the 1940s that inositol lipids are highly metabolically active and, more recently, that they are components of a phosphoinositide-mediated signal transduction system. The concentration of $\mathrm{mI}$ in neural cells is about 2 to 500 times that of plasma - a gradient for which a high affinity sodium-mI transporter (SMIT) is responsible. SMIT mRNA and protein expression are highest in the fetal brain, a fact reflected in the high concentration of $\mathrm{mI}$ in fetal CSF (21) and in the newborn brain (8). In fact, $\mathrm{mI}$ is the largest peak seen in proton spectra from normal newborn brain (22). A second $\mathrm{pH}$ sensitive mam- malian $\mathrm{mI}$ transporter (H+/myo-inositol symporter; HMIT) has also recently been identified (23). In addition to synthesis and active transport of $\mathrm{mI}$, a third primary method of cellular $\mathrm{mI}$ regulation is facilitated diffusion, responsible for $\mathrm{mI}$ efflux in neurons and glia (7). It should also be noted that $\mathrm{mI}$ seen spectroscopically represents free rather than bound intracellular $\mathrm{mI}$ rather than extracellular $\mathrm{mI}(24)$.

Myoinositol and previous MRS studies. Several previous investigations in adults have reported on $\mathrm{mI}$ ratios after TBI. In one study of 26 patients, scanned 12 d postinjury, MRS from normal appearing posterior frontal white matter had increased $\mathrm{mI} / \mathrm{Cre}$ ( $\mathrm{mI}$ reported as Ins) as well as decreased NAA/Cre and increased Cho/Cre (25). The increases in $\mathrm{mI}$ and Cho observed in this study were considered reflective of glial cell proliferation. A second study reported spectral data of serial MRS

Table 3. Spearman Rho correlation coefficients and $p$ values for selected clinical and spectroscopy values for OGM and PWM quantitative MI values

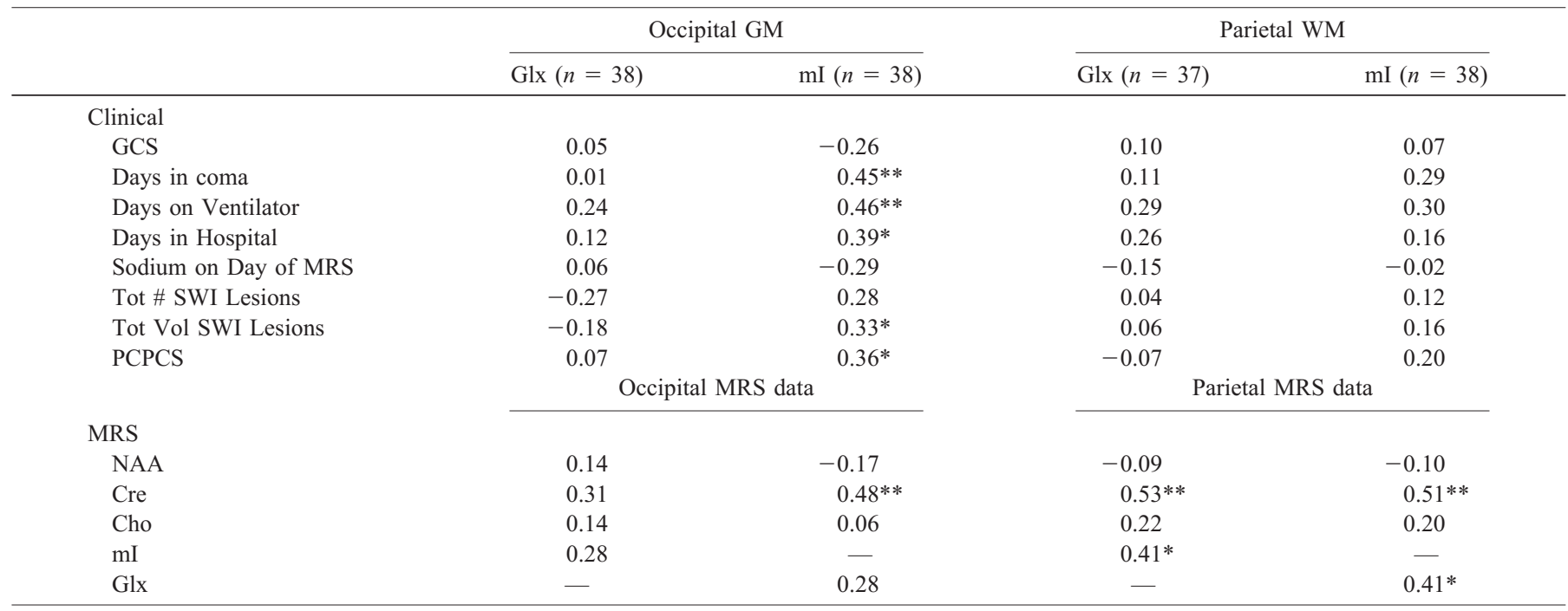

${ }^{*} p<0.05 ;{ }^{* *} p<0.01$. 
Table 4. $O G M$ and PWM quantitative MI values

\begin{tabular}{|c|c|c|c|c|c|}
\hline Variable & $\begin{array}{c}\% \\
\text { predicted }\end{array}$ & $\begin{array}{c}\text { \# of false } \\
\text { positives }\end{array}$ & $\begin{array}{l}\text { \# of false } \\
\text { negatives }\end{array}$ & $\chi^{2}$ & $p$ value \\
\hline \multicolumn{6}{|l|}{ Clinical } \\
\hline GCS & $76 \%$ & $0 / 29$ & $9 / 9$ & 4.2 & 0.52 \\
\hline GCS, \# days coma, \# days on vent. & $82 \%$ & $1 / 29$ & $6 / 9$ & 3.6 & 0.89 \\
\hline \multicolumn{6}{|l|}{ SWI } \\
\hline SWI number and volume & $79 \%$ & $2 / 29$ & $6 / 9$ & 9.9 & 0.27 \\
\hline OGM Glx & $79 \%$ & $0 / 29$ & $8 / 9$ & 13.0 & 0.11 \\
\hline OGM NAA & $76 \%$ & $0 / 29$ & $9 / 9$ & 5.6 & 0.70 \\
\hline OGM Cre & $79 \%$ & $1 / 29$ & $7 / 9$ & 8.4 & 0.39 \\
\hline OGM Cre, mI & $82 \%$ & $2 / 29$ & $5 / 9$ & 8.8 & 0.36 \\
\hline OGM Cre, mI, Glx & $82 \%$ & $2 / 29$ & $5 / 9$ & 14.0 & 0.08 \\
\hline PWM NAA & $82 \%$ & $1 / 29$ & $6 / 9$ & 4.0 & 0.78 \\
\hline OGM mI, Cre, PWM NAA, SWI \# & $87 \%$ & $1 / 29$ & $4 / 9$ & 3.9 & 0.87 \\
\hline
\end{tabular}

GCS, Glasgow Coma Scale score; SWI, susceptibility weighted imaging; OGM, occipital gray matter; NAA, n-acetyl aspartate; Cre, creatine; Cho, choline; $\mathrm{mI}$, myoinositol; PWM, parietal white matter.

$\chi^{2}$ obtained with Hosmer-Lemeshow Goodness of fit test.

examinations in 19 adult patients at 1.5, 3, and 6 mo (3). Myoinositol (reported as mIs) in gray matter was increased at 6 mo compared with controls whereas $\mathrm{mI}$ in white matter was increased at 1.5 and 6 mo. Cognitive function testing, done at the time of MRS, showed a correlation at 1.5 mo between $\mathrm{mI}$ and cognitive outcome but not at 3 or 6 mo. Studies of $\mathrm{mI}$ after pediatric TBI have not been previously reported.

The results of the logistic regression analysis demonstrate that in children as in adults, MRS variables are predictive of outcome. In particular, MRS variables used alone or in combination resulted in correctly predicting outcome in 79 to $82 \%$ of the cases. This was similar to clinical and neuroimaging variables, however, only the results using MRS variables approached significance. Our results show that one can improve the ability to predict outcome after serious TBI in children $(5,6)$ and in the present study, mI was useful for this purpose.

Astrogliosis. The reason for the increase in $\mathrm{mI}$ observed in our patients is unknown but astrogliosis is suspected. It is well known that astrogliosis (increased astrocyte number and activity) begins soon after TBI and can persist for weeks to months (9). Several previous MRS studies have supported the idea that increases in $\mathrm{mI}$ reflect an increase in glial content or glial proliferation $(2,3,26)$. Reports in patients with multiple sclerosis during the acute stages of demyelination due to the accumulation of myelin breakdown products as well as in chronic inactivated demyelinated lesions have revealed elevated $\mathrm{mI}$ attributed to astrocytosis (27). Although the pathophysiology of multiple sclerosis differs from TBI, these findings lend indirect support to our observation that the increase in $\mathrm{mI}$ in our patients was due to a combination of acute accumulation of DAI-associated myelin breakdown products and the later development of astrocytosis.

More recent studies in a rat brain contusion model also support our belief that increased $\mathrm{mI}$ after $\mathrm{TBI}$ is due to astrogliosis. Although $\mathrm{mI}$ decreased initially in these experi- mental studies, it was increased by $31 \%$ seven days after injury, the same time frame that our MRS studies were performed, although the time frame in animal models may be different from that observed in human TBI (28). These findings in the rat brain correlated with a substantial increase in two markers of astrocytic activity: a diffuse increase in glial fibrillary acidic protein immunohistochemical staining and an increase in the number of vimentin-positive astrocytes. These findings also were correlated with an increase in choline and were interpreted as a reflection of glial activation.

Of interest is that in our study, outcome correlated with OGM but not with PWM mI levels (Table 2). In one report in adults, $\mathrm{mI}$ was greater in PWM but not OGM at 1.5 mo but by 6 mo both regions showed increased $\mathrm{mI}$ compared with controls (3). Another study also looked at early (i.e., 12 d) versus. late $(6 \mathrm{mo}) \mathrm{mI}$ data but only in frontal white matter and found increased $\mathrm{mI}(25)$. The fact that the majority of astrocytes are located near cortical gray matter neurons, rather than in white matter, may explain why we found that outcome correlated with OGM mI levels but not with PWM mI levels. As our spectra were obtained from occipital gray matter $7 \mathrm{~d}$ (range 1-17 d) postinjury, the differences between our results and those in adult TBI patients suggest the need for additional studies to determine whether these differences are age or time dependent.

If posttraumatic astrogliosis interferes with neural repair mechanisms and axonal regeneration, the presence of elevated $\mathrm{mI}$ as a marker of astrogliosis may be clinically important as a variety of experimental approaches to reduce astrogliosis are currently under investigation and eventually could be applied to patients. These include astrocyte ablation, transgenic approaches to reduce extracellular matrix formation, attenuation of factors stimulating astrogliosis, inhibition of mediators of the posttraumatic inflammatory response, gene manipulation of astrocyte function, etc (9). 
Osmolyte regulation. A second reason for increased $\mathrm{mI}$ may relate to its role as an osmolyte. Cerebral edema is common after TBI and DAI and can be vasogenic, due to breakdown of the blood-brain barrier to macromolecules, and cytotoxic, due to disturbed cell metabolism. Disruption of the blood brain barrier leads to extravasation of proteins and other osmotically active molecules. As a protective response, $\mathrm{mI}$ levels are increased by several mechanisms, including the up-regulation of SMIT mRNA (29). Separately, cytotoxic injury can occur after TBI, as is commonly associated with focal ischemia and stroke. Myoinositol transport has been shown to be diminished after stroke (30). A rise in intracellular electrolytes and accumulation of osmotically active catabolites would normally be balanced by $\mathrm{mI}$ efflux. Because of diminished ATP, required for the volume-sensitive organic osmolyte anion channel, $\mathrm{mI}$ efflux is diminished and results in neuronal swelling $(7,31)$. Neuropathological studies have also found that diffuse edema is common in patients with DAI (32).

In our patients, the role of $\mathrm{mI}$ in osmotic balance may have been influenced by the administration of hypertonic saline for treatment of increased ICP. In the face of hypernatremia the brain accumulates $\mathrm{mI}$ to decrease water loss $(7,33,34,35)$. In addition, if hypernatremia is corrected too rapidly, the sudden change in osmotic gradient may lead to massive cerebral edema. Of our 38 patients, 21 had ICP monitoring based on the severity of their injury and initial neuroimaging findings. Of these individuals; 17 had elevated ICP. Although 27 of our 38 patients received hypertonic 3\% normal saline to control ICP or for other clinical reasons (e.g. pupillary changes in patients who were not being monitored), we found no correlation between serum sodium levels, either initially or on the day of MRS, with either OGM or PWM mI levels (Table 3). We also did not observe any correlation between ICP and OGM or PWM mI levels.

Glutamate. In one recent study of children with different forms of TBI, early measurement of CSF glutamate was found increased in children less than four years of age primarily with nonaccidental trauma and correlated with outcome (14). Older children with TBI due to pedestrian versus motor vehicle accidents did not show a consistent increase in glutamate nor was there a correlation with outcome. A trend toward an association between high glutamate concentrations and ischemic blood flow was also observed. The Glx peak, which is a combination of glutamate, glutamine and GABA (gamma-amino-butyric acid), was increased in all of our TBI patients, not just those with poor outcome. Although Glx levels did not correlate with outcome, they did correlate with the mI levels.

Glutamate may increase cell swelling and presumably this may also increase $\mathrm{mI}$ (36). In the current study, MRS was performed on average $7 \mathrm{~d}$ after injury, suggesting that there may be prolonged glutamate release which may contribute to the severity of injury by several mechanisms and in part could be mediated by glutamate induced increases in mI. Glutamate increases, if extracellular, may reflect greater primary axonal injury and transmitter leakage or greater secondary neuronal and glial energy failure and free-radical injury, with neuronal depolarization, $\mathrm{Ca}++$ increases, or decreased glial transmitter reuptake.
Overall the findings from out study and those involving CSF glutamate measurements suggest that the role of glutamate after TBI in children remains unclear and could be affected by the age of the patient, the nature and severity of injury, the association of secondary ischemic injury and presumably other variables.

Other mechanisms. Intracellular calcium is also important in the regulation of $\mathrm{mI}$ content (7). Recent investigations in a stretch-injured astrocyte model has found that such injury results in a rapid increase of intracellular $\mathrm{Ca}^{++}$and also that intracellular calcium stores were no longer able to release $\mathrm{Ca}^{++}$in response to inositol triphosphate (IP3) (37). This suggested that IP3 uncoupling might contribute to pathologic alterations and further brain injury. In our patient population, with the degree of DAI that we observed, stretch injury likely occurred and could have resulted in increased intracellular $\mathrm{Ca}^{++}$. This may be another mechanism that contributed to the increase in $\mathrm{mI}$ and the correlation between $\mathrm{mI}$ and the number and volume of SWI lesions.

\section{CONCLUSIONS}

The finding that $\mathrm{mI}$ is increased in children with TBI and that it correlates with long-term outcome is intriguing. It may serve as a marker for posttraumatic astrogliosis or perhaps reflect disturbances in osmotic regulation. In either case, spectroscopic monitoring of $\mathrm{mI}$ in patients with TBI may be helpful and potentially could be used as a biochemical marker of the development of astrogliosis and of treatment.

\section{REFERENCES}

1. Brooks WM, Friedman SD, Gasparovic C 2001 Magnetic resonance spectroscopy in traumatic brain injury. J Head Trauma Rehabil 16:146-164

2. Ross BD, Ernst T, Kreis R, Haseler LJ, Bayer S, Danielsen E, Bluml S, Shonk T, Mandigo JC, Caton W, Clark C, Jensen SW, Lehman NL, Arcinue E, Pudenz R, Shelden CH 1998 1H MRS in acute traumatic brain injury. J Magn Reson Imaging $8: 829-840$

3. Brooks WM, Stidley CA, Petropoulos H, Jung RE, Weers DC, Friedman SD, Barlow MA, Sibbitt WL Jr., Yeo RA 2000 Metabolic and cognitive response to human traumatic brain injury: a quantitative proton magnetic resonance study. J Neurotrauma 8:629-640

4. Friedman SF, Brooks WM, Jung RE, Chiulli SJ, Sloan JH, Montoya BT, Hart BL, Yeo RA 1999 Quantitative proton MRS predicts outcome after traumatic brain injury. Neurology 52:1384-1391

5. Ashwal S, Holshouser BA, Shu SK, Simmons PL, Perkin RM, Tomasi LG, Knierim DS, Sheridan C, Craig K, Andrews GH, Hinshaw DB 2000 Predictive value of proton MR spectroscopy in pediatric closed head injury. Pediatr Neurol 23:114-125

6. Brenner T, Freier MC, Holshouser BA, Burley T, Ashwal S 2003 Predicting neuropsychologic outcome after traumatic brain injury in children. Pediatr Neurol 28:104114

7. Fisher SK, Novak JE, Agranoff BW 2002 Inositol and higher inositol phosphates in neural tissues: homeostasis, metabolism and functional significance. $\mathrm{J}$ Neurochem $82: 736-754$

8. Robertson NJ, Lewis RH, Cowan FM, Allsop JM, Counsell SJ, Edwards AD, Cox IJ 2001 Early increases in brain myo-inositol measured by proton magnetic resonance spectroscopy in term infants with neonatal encephalopathy. Pediatr Res 50:692-700

9. McGraw J, Hiebert GW, Steeves JD 2001 Modulating astrogliosis after neurotrauma J Neurosci Res 63:109-115

10. Rostworowski M, Balasingam V, Chabot S, Owens T, Yong VW 1997 Astrogliosis in the neonatal and adult murine brain post-trauma: elevation of inflammatory cytokines and the lack of requirement for endogenous interferon-gamma. J Neurosci $17: 3664-3674$

11. Tong KA, Ashwal S, Holshouser BA, Shutter LA, Herigault G, Haacke EM, Kido DK 2003 Hemorrhagic shearing lesions in children and adolescents with posttraumatic diffuse axonal injury: improved detection and initial results. Radiology 227:332339

12. Bullock R, Zauner A, Woodward JJ, Myseros J, Choi SC, Ward JD, Marmarou A, Young HF 1998 Factors affecting excitatory amino acid release following severe human head injury. J Neurosurg 89:507-518 
13. Koura SS, Doppenberg EM, Marmarou A, Choi S, Young HF, Bullock R 1998 Relationship between excitatory amino acid release and outcome after severe human head injury. Acta Neurochir Suppl (Wien) 71:244-246

14. Ruppel RA, Kochanek PM, Adelson PD, Rose ME, Wisniewski SR, Bell MJ, Clark RS, Marion DW, Graham SH 2001 Excitatory amino acid concentrations in ventricular cerebrospinal fluid after severe traumatic brain injury in infants and children: the role of child abuse. J Pediatr 138:18-25

15. Fiser DH 1992 Assessing the outcome of pediatric intensive care. J Pediatr 121:68-74

16. Fiser DH, Long N, Roberson PK, Hefley G, Zolten K, Brodie-Fowler M 2000 Relationship of pediatric overall performance category and pediatric cerebral performance category scores at pediatric intensive care unit discharge with outcome measures collected at hospital discharge and 1- and 6-month follow-up assessments. Crit Care Med 28:2616-2620

17. Reichenbach JR, Venkatesan R, Schillinger DJ, Kido DK, Haacke EM 1997 Smal vessels in the human brain: MR venography with deoxyhemoglobin as an intrinsic contrast agent. Radiology 204:272-277

18. Holshouser BA, Ashwal S, Luh GY, Shu S, Kahlon S, Auld KL, Tomasi LG, Perkin RM, Hinshaw DB Jr 1997 Proton MR spectroscopy after acute central nervous system injury: outcome prediction in neonates, infants and children. Radiology 202:487-496

19. Provencher SW 1993 Estimation of metabolite concentrations from localized in vivo proton NMR spectra. Magn Reson Med 30:672-679

20. Provencher SW 2001 Automatic quantitation of localized in vivo $1 \mathrm{H}$ spectra with LCModel. NMR Biomed 14:260-264

21. Battaglia FC, Meschia G, Blechner JN, Barron DH 1961 The free myo-inositol concentration of adult and fetal tissues of several species. Q J Exp Physiol Cogn Med Sci 46:188-193

22. Danielsen ER, Ross B 1999 Magnetic Resonance Spectroscopy Diagnosis of Neurological Diseases. Marcel Dekker, New York, p 349

23. Uldry M, Ibberson M, Horisberger JD, Chatton JY, Riederer BM, Thorens B 2001 Identification of a mammalian $\mathrm{H}(+)$-myo-inositol symporter expressed predominantly in the brain. EMBO J 20:4467-4477

24. Frahm J, Hanefeld F 1997 Localized proton magnetic spectroscopy of brain disorders in childhood. In: Bachelard H (ed) Magnetic Resonance Spectroscopy and Imaging in Neurochemistry. Plenum Press, New York, p 329-402

25. Garnett MR, Blamire AM, Corkill RG, Cadoux-Hudson TA, Rajagopalan B, Styles P 2000 Early proton magnetic resonance spectroscopy in normal-appearing brain correlates with outcome in patients following traumatic brain injury. Brain 123:20462054

26. Chang L, Ernst T, Leonido-Yee M, Witt M, Speck O, Walot I, Miller EN 1999 Highly active antiretroviral therapy reverses brain metabolite abnormalities in mild HIV dementia. Neurology 53:782-789

27. Bitsch A, Bruhn H, Vougioukas V, Stringaris A, Lassman H, Frahm J, Bruck W 1999 Inflammatory CNS demyelination: histopathologic correlation with in vivo quantitative proton MR spectroscopy. AJNR Am J Neuroradiol 20:1619-1627

28. Schuhmann MU, Stiller D, Skardelly M, Bernarding J, Klinge PM, Samii A, Samii M, Brinker T 2003 Metabolic changes in the vicinity of brain contusions: a proton magnetic resonance spectroscopy and histology study. J Neurotrauma 20:725-743

29. Yamashita T, Shimada S, Yamauchi A, Guo W, Kohmura E, Hayakawa T, Tohyama M 1997 Induction of $\mathrm{Na}+$ /myo-inositol co-transporter mRNA after rat cryogenic injury. Brain Res Mol Brain Res 46:236-242

30. Venkatachalam MA, Buja M 1994 Cellular injury and cellular death. In: Cotran RS Kumar V, Robbins SL, Schoen FJ, (eds) Robbins Pathologic Basis of Disease, $5^{\text {th }}$ ed, Philadelphia: WB Saunders, pp 1-34

31. Jackson PS, Morrison R, Strange K 1994 The volume-sensitive organic osmolyteanion channel VSOAC is regulated by nonhydrolytic ATP binding. Am J Physiol 267(5 Pt 1):C1203-C1209

32. Onaya M 2002 Neuropathological investigation of cerebral white matter lesions caused by closed head injury. Neuropathology 22:243-251

33. Lohr JW, McReynolds J, Grimaldi T, Acara M 1988 Effect of acute and chronic hypernatremia on myoinositol and sorbitol concentration in rat brain and kidney. Life Sci 43:271-276

34. Lien YH, Shapiro JI, Chan L 1990 Effects of hypernatremia on organic brain osmoles. $\mathrm{J}$ Clin Invest 85:1427-1435

35. Lee JH, Arcinue E, Ross BD 1994 Brief report: organic osmolytes in the brain of an infant with hypernatremia. N Engl J Med 331:439-442

36. Staub F, Peters J, Plesnila N, Chang RC, Baethmann A 1997 Swelling and damage of glial cells by lactacidosis and glutamate: effect of alpha-trinositol. Brain Res 766:285-288

37. Floyd CL, Rzigalinski BA, Weber JT, Sitterding HA, Willoughby KA, Ellis EF 2001 Traumatic injury of cultured astrocytes alters inositol (1,4,5)-trisphosphate-mediated signaling. Glia 33:12-23 